\title{
Conditional detection of pure quantum states of light after storage in a waveguide
}

\author{
Erhan Saglamyurek, ${ }^{1, \text { f }}$ Neil Sinclair, ${ }^{1, \text { * }}$ Jeongwan Jin,, , * Joshua A. Slater, ${ }^{1,}$, Daniel Oblak, ${ }^{1}$ \\ Félix Bussières, ${ }^{1}$ † Mathew George,${ }^{2}$ Raimund Ricken, ${ }^{2}$ Wolfgang Sohler, ${ }^{2}$ and Wolfgang Tittel ${ }^{1}$ \\ ${ }^{1}$ Institute for Quantum Information Science, and Department of Physics 85 Astronomy, \\ University of Calgary, 2500 University Drive NW, Calgary, Alberta T2N 1N4, Canada \\ ${ }^{2}$ Department of Physics - Applied Physics, University of Paderborn, \\ Warburger Str. 100, 33095 Paderborn, Germany
}

\begin{abstract}
Conditional detection is an important tool to extract weak signals from a noisy background and is closely linked to heralding, which is an essential component of protocols for long distance quantum communication and distributed quantum information processing in quantum networks. Here we demonstrate the conditional detection of time-bin qubits after storage in and retrieval from a photon-echo based waveguide quantum memory. Each qubit is encoded into one member of a photon-pair produced via spontaneous parametric down conversion, and the conditioning is achieved by the detection of the other member of the pair. Performing projection measurements with the stored and retrieved photons onto different bases we obtain an average storage fidelity of $0.885 \pm 0.020$, which exceeds the relevant classical bounds and shows the suitability of our integrated light-matter interface for future applications of quantum information processing.
\end{abstract}

PACS numbers: 03.67.Hk, 42.50.Ex, 32.80.Qk, 78.47.jf

Quantum memories are key elements for future applications of quantum information science such as long-distance quantum communication via quantum repeaters [1, 2] and, more generally, distributed quantum information processing in quantum networks [3]. They enable reversible mapping of arbitrary quantum states between travelling and stationary carriers (i.e. light and matter). This reduces the impact of loss on the time required to establish entanglement between distant locations [1, and allows the implementation of local quantum computers based on linear optics 44. However, towards these ends, the successful transfer of a quantum state into the memory must be announced by a heralding signal. When using an individual absorber, such a signal can be derived through the detection of a change of atomic level population [5]. In atomic ensembles, this approach is infeasible. Instead, storage is derived from the detection of a second photon that either indicates the absorption [6], or the presence of the first at the input of the memory 7 ] (the first approach relies on spontaneous Raman scattering, the second on using pairs of photons). Furthermore, quantum memories must allow on-demand read-out after second-long storage with high efficiency [7, 8], and, for viable quantum technology, should be robust and simple to operate (e.g. be based on integrated optics).

A lot of progress towards these (and other) figures of merit has been reported over the past few years, including work that explores electromagnetically induced transparency (EIT), as well as photon-echo and cavity QEDbased approaches (see [2, 5, 7, 14] for reviews and latest achievements). Yet, strictly, most of these experiments did not report true heralding - either heralding was not actually implemented, or the 'heralding' signal was generated only after the stored photon left the memory, or the signal could, due to technical issues, only be derived once the stored photon was detected. Nevertheless, experiments that employ photon pairs [11 13, 15] do gain from conditioning the detection of the stored photon on that of the auxiliary photon (i.e. a posteriori 'heralding'): by reducing the effects of loss and detector noise conditioning generally increases the fidelity between the quantum state of the original and the retrieved photon.

Supplementing the experiments on storage of entangled photons [11 13, 15, we now report another step towards the goal of building universal, viable, and heralded quantum memory devices - the storage of photons in pure quantum states in a solid state waveguide, their retrieval, and their conditional detection by means of temporal correlations with auxiliary photons. We point out that the step to true heralding is minor and of purely technical nature; it simply requires using different, existing, singlephoton detectors (see, e.g., [16, 17]).

Our experimental setup consists of two main blocks, see Fig. 1. A spontaneous parametric down-conversion (SPDC) photon-pair source, and a Ti:Tm:LiNbO 3 single mode waveguide fabricated by indiffusion processes [18. When cooled to $3 \mathrm{~K}$, and using a photon-echo quantum memory protocol [7, 8, 19, the Tm-doped waveguide allows storage and retrieval of quantum states encoded into one member of each photon pair, while the detection of the other member provides the conditioning signal.

In the photon-pair source a mode-locked pump laser generates 6 ps long pulses at a rate of $80 \mathrm{MHz}$ and $1047.328 \mathrm{~nm}$ central wavelength. They are subsequently frequency-doubled (FD) in a periodically poled $\mathrm{LiNbO}_{3}$ (PPLN) crystal, yielding pulses with $523.664 \mathrm{~nm}$ central wavelength, $16 \mathrm{ps}$ duration, and $90 \mathrm{~mW}$ average power. The FD pulses are sent to a second PPLN crystal that, via SPDC, produces pairs of photons centred at $795.506 \mathrm{~nm}$ and $1532.426 \mathrm{~nm}$. Frequency filtering 


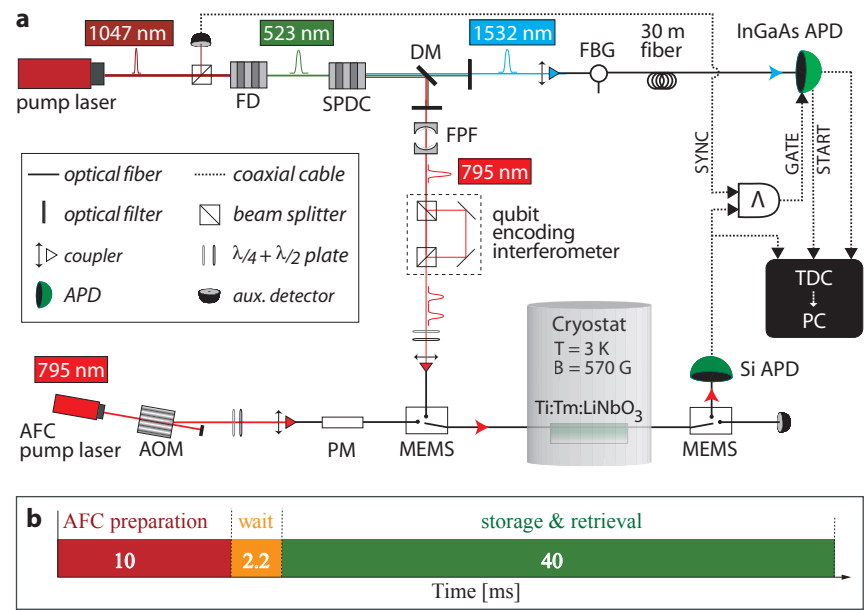

FIG. 1: (color online) a. Photon pair source and quantum memory setup (see text for details). Wave-plates align light polarization along the $\mathrm{LiNbO}_{3}$ 's $\mathrm{C}_{3}$-axis. The waveguide is held at $3 \mathrm{~K}$, and a $570 \mathrm{G}$ magnetic field is applied along the crystal's $\mathrm{C}_{3}$-axis (see Fig. 2a). b. Timing sequence containing three repeated phases: $10 \mathrm{~ms} A F C$ preparation for optical pumping, $2.2 \mathrm{~ms}$ wait to allow excited population to decay, and $40 \mathrm{~ms}$ storage and retrieval, during which $795 \mathrm{~nm}$ photons are successively stored for $t_{s t}=6 \mathrm{~ns}$ and then recalled.

the $795 \mathrm{~nm}$ photons with a $6 \mathrm{GHz}$-bandwidth FabryPerot filter (FPF) and the $1532 \mathrm{~nm}$ photons with a $9 \mathrm{GHz}$-bandwidth fiber-Bragg grating (FBG) we obtain frequency uncorrelated pairs. Each $795 \mathrm{~nm}$ photon travels through an imbalanced Mach-Zehnder interferometer with $42 \mathrm{~cm}$ path-length difference, corresponding to 1.4 ns relative delay. Thus, each photon emerges in a superposition of two temporal modes (early and late), i.e., in a time-bin qubit state [20]. They are then directed into the quantum memory, stored, retrieved, and finally detected by a $\mathrm{Si}$ avalanche-photo-diode (APD)based single-photon detector.

All $1532 \mathrm{~nm}$ photons are sent through $30 \mathrm{~m}$ standard telecommunication fiber to an InGaAs APD-based singlephoton detector. As is typically done, the detector is gated to reduce noise. The gate signal could in principle be the SYNC signal derived from each pulse emitted by the pump laser. However, as its repetition rate of $80 \mathrm{MHz}$ by far exceeds the maximum gate frequency of our detector, around $1 \mathrm{MHz}$, we first AND the SYNC pulses with pulses generated by each Si-APD detection, and then use this low-rate signal to gate the InGaAsAPD. Provided the latter is ready for photon detection (i.e. not deadtime-blocked due to a previous detection), this signal also starts a time-to-digital converter (TDC), which then records the time-difference between the detection events produced by the Si-APD and the InGaAsAPD. This data is used to obtain statistics for single detections of the retrieved $795 \mathrm{~nm}$ photons, as well as for detections conditioned on the existence of $1532 \mathrm{~nm}$ pho- tons. We emphasize that if an InGaAs APD supporting $80 \mathrm{MHz}$ gate rate had been available [16, 17, then 1532 $\mathrm{nm}$ photons could have been detected without the need for a priori detection of a $795 \mathrm{~nm}$ photon. This simple modification of our setup would have turned the conditional detection of $795 \mathrm{~nm}$ photons into detections that are heralded by clicks of the InGaAs APD.

The other main block of our setup is a Ti:Tm:LiNbO 3 waveguide that allows storage and retrieval of the $795 \mathrm{~nm}$ photons via the atomic frequency comb (AFC) quantum memory protocol [19. This approach to quantum state storage requires the spectral absorption of an atomic ensemble to be constituted of a series of equally spaced lines with frequency spacing $\Delta_{\nu}$. The interaction between such an $\mathrm{AFC}$ and a photon with wavevector $k$ leads to the absorption of the photon and generates a collective excitation in the atomic medium that is described by

$$
|\Psi\rangle=\frac{1}{\sqrt{N !}} \sum_{j=1}^{N} c_{j} e^{i 2 \pi m_{j} \Delta_{\nu} t} e^{-i k z_{j}}\left|g_{1}, \cdots e_{j}, \cdots g_{N}\right\rangle .
$$

Here, $\left|g_{j}\right\rangle\left(\left|e_{j}\right\rangle\right)$ denotes the ground (excited) state of atom $j, m_{j} \Delta_{\nu}$ is the detuning of the atom's transition frequency from the photon carrier frequency, $z_{j}$ its position measured along the propagation direction of the light, and the factor $c_{j}$ depends on the atom's resonance frequency and position. Due to the presence of different atomic transition frequencies, the excited collective coherence dephases rapidly. However, the particular shape of the absorption line results in the recovery of the collective coherence after storage time $t_{s t}=1 / \Delta_{\nu}$. This can easily be seen from Eq. (1): for $t=1 / \Delta_{\nu}$ all frequency dependent phase factors are zero $(\bmod 2 \pi)$. This leads to re-emission of the photon into the original mode and quantum state with maximally $54 \%$ efficiency for an optimally implemented AFC. Modifications to the procedure enable recall on demand and up to $100 \%$ efficiency [19].

Suitable media in which to implement the AFC protocol are cryogenically cooled rare-earth ion doped crystals [7, 21]. They feature inhomogeneously broadened absorption profiles, often posses long-lived atomic sublevels that can serve as shelving levels for tailoring the AFC through persistent spectral hole burning, and generally have long coherence times on optical and spin transitions. We use the ${ }^{3} \mathrm{H}_{6}{ }^{3} \mathrm{H}_{4}$ transition of Tm ions in a single-mode channel waveguide fabricated by $\mathrm{Ti}$ indiffusion into the Tm doped surface of a Z-cut $\mathrm{LiNbO}_{3}$ crystal, see Fig. 2a [18. To tailor the desired AFC into the inhomogeneously broadened absorption profile, Tm ions with transition frequencies within the comb's troughs are optically pumped via the excited level into long-lived nuclear Zeeman levels, see Fig. 2b [18, 22]. To achieve frequency selective optical pumping we employed a linear side-band chirp technique [11, 23] that allowed us to create a $5 \mathrm{GHz}$ broad grating (matching the spectral width of the $795 \mathrm{~nm}$ photons) with tooth spacing of $167 \mathrm{MHz}$, 
a

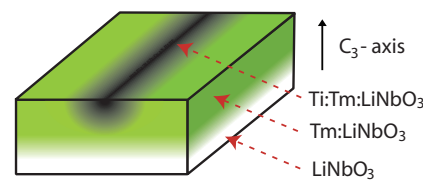

c

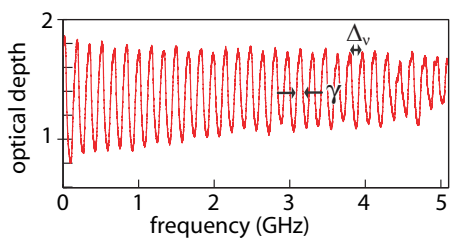

b

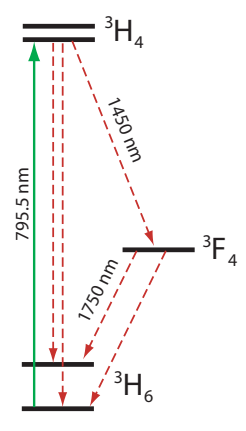

FIG. 2: (color online) a. Waveguide geometry: The sample surface is first doped by indiffusing a $\approx 20 \mathrm{~nm}$ thick Tm layer yielding a concentration profile of $\approx 6 \mu \mathrm{m}$ depth with $\approx 10^{20}$ ions per $\mathrm{cm}^{3}$ surface concentration. Subsequently a $3 \mu \mathrm{m}$ wide channel waveguide is fabricated by indiffusion of a $40 \mathrm{~nm}$ thick vacuum-deposited Ti stripe. AFC preparation light and single photons are coupled in and out of the waveguide with $10 \%$ total efficiency by butt-coupling single mode fibers. b. Simplified energy level diagram of Tm ions: The optical coherence time of the ${ }^{3} \mathrm{H}_{6}{ }^{3} \mathrm{H}_{4}$ transition at $3 \mathrm{~K}$ is $1.6 \mu \mathrm{s}$, and the radiative lifetimes of the ${ }^{3} \mathrm{H}_{4}$ and ${ }^{3} \mathrm{~F}_{4}$ levels are $82 \mu \mathrm{s}$ and $2.4 \mathrm{~ms}$, respectively. A $570 \mathrm{G}$ magnetic field splits the ground and excited levels into Zeeman sub-levels. The ground Zeeman level splitting is $\sim 83 \mathrm{MHz}$, and the lifetime of the upper ground level exceeds one second. c. $5 \mathrm{GHz}$-bandwidth AFC: The tooth separation is $\Delta_{\nu}=167 \mathrm{MHz}$, corresponding to $6 \mathrm{~ns}$ storage time. The line-width of the teeth is $\gamma=83 \mathrm{MHz}$.

see Fig. 22. This corresponds to a storage time of $6 \mathrm{~ns}$. After each 10 ms-long AFC preparation a $2.2 \mathrm{~ms}-$ long wait time allows atoms excited by the optical pumping to decay before the photon storage (see Fig. 1 $\mathrm{b}$ for the timing per experimental cycle). A set of micro electromechanical switches (MEMS) then open the channel for qubits to enter the memory, and, after recall, direct them towards the Si-APD. We assessed our memory's retrieval efficiency to be $2 \%$. Taking the $10 \mathrm{~dB}$ fibre-to-fibre coupling loss in and out of the waveguide into account, this yields an overall system efficiency of $0.2 \%$.

An interesting and useful aspect of photon-echo quantum memory protocols is that they provide a robust tool to manipulate time-bin qubits 24 27. For example, using the AFC approach, any projection measurement on time-bin qubit states can be performed by superimposing two combs (double AFC) with appropriately chosen relative center frequencies and amplitudes [25. This leads to two re-emission times that can be set to differ by the temporal mode separation of the qubit to be analyzed (1.4 ns for our experiments). Hence, as a previously absorbed photon is re-emitted by the superimposed combs, early and late temporal modes interfere, allowing the qubit state to be analyzed in the same way as is typically done with an imbalanced Mach-Zehnder interferometer 25. Double AFC recall will, however, lead to a reduction of the recall efficiency (compared to single recall).
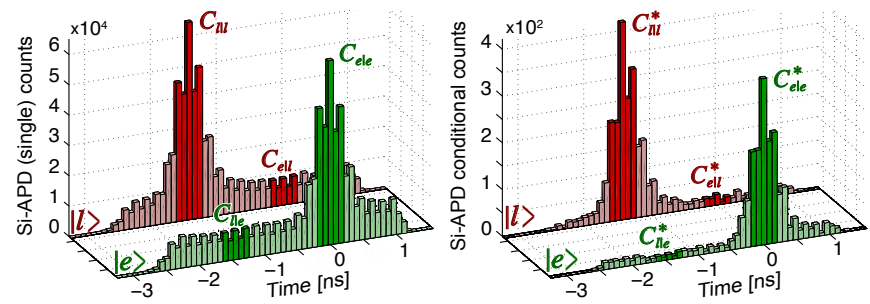

FIG. 3: (color online) Storage of early and late time-bin qubit states in the AFC memory. The left-hand figure depicts the histograms from 180 min of single detections of the retrieved $795 \mathrm{~nm}$ photons prepared in early (red) and late (green) qubit states with the highlighted regions marking the relevant detection windows. The right-hand figure shows the detections conditioned on $1532 \mathrm{~nm}$ photons for the same states. Without conditioning the fidelities are $\mathcal{F}_{e}=0.8652 \pm 0.0006$ and $\mathcal{F}_{l}=0.8376 \pm 0.0004$ for the storage of early and late time-bin states, respectively. Correspondingly, with conditioning, the fidelities are $\mathcal{F}_{e}^{*}=0.9505 \pm 0.0058$ and $\mathcal{F}_{l}^{*}=0.9573 \pm 0.0033$.

To demonstrate faithful storage and retrieval of quantum states from the memory, we performed projection measurements with various time-bin qubits onto different bases using single (standard) and double AFC schemes as explained before. In all our measurements the average photon number per qubit was 0.1 at the output of the qubit-preparation interferometer. First we generated qubit states that occupy only early $|e\rangle$ or late $|l\rangle$ temporal modes by blocking either the long or short arm of the qubit-encoding interferometer, respectively, and then stored these states in the memory for 6 ns. Fig. 3 (left) shows single detections (no conditioning) of the retrieved photons as a function of the time difference with respect to the START signal. The dark counts from the $\mathrm{Si}-\mathrm{APD}$ reduce the signal to noise ratio (SNR) to $\sim 5$. For an input state $|e\rangle$, we compute the fidelity as $\mathcal{F}_{e}=C_{e \mid e} /\left(C_{e \mid e}+C_{l \mid e}\right)$, where, e.g., $C_{l \mid e}$ denotes the number of detected counts in the late time-bin given $|e\rangle$ was encoded in the qubit at the input. Similarly, we can find $\mathcal{F}_{l}$, enabling us to calculate the mean fidelity: $\mathcal{F}_{e l}=\left(\mathcal{F}_{e}+\mathcal{F}_{l}\right) / 2=0.8514 \pm 0.0004$. On the other hand, conditioning the detections of the retrieved photons on the detection of $1532 \mathrm{~nm}$ photons leads to a substantial increase of the SNR to $\sim 22$, as shown in Fig. 3 (right). This yields a mean fidelity of $\mathcal{F}_{e l}^{*}=0.954 \pm 0.015$.

Next, qubit states in an equal superposition of early and late temporal modes $\frac{1}{\sqrt{2}}\left(|e\rangle+e^{i \phi}|l\rangle\right)$ were produced with $\phi$ set to zero. Storage and projection measurements were performed using the double AFC scheme with the relative phase of the two combs (measured w.r.t. the phase introduced by the qubit-preparation interferometer) varied by $\pi / 2$ increments. The results for single and conditional detections are given in Fig. 4. The histograms show the detection statistics for zero and $\pi$ double AFC phase settings, from which we extract a SNR 

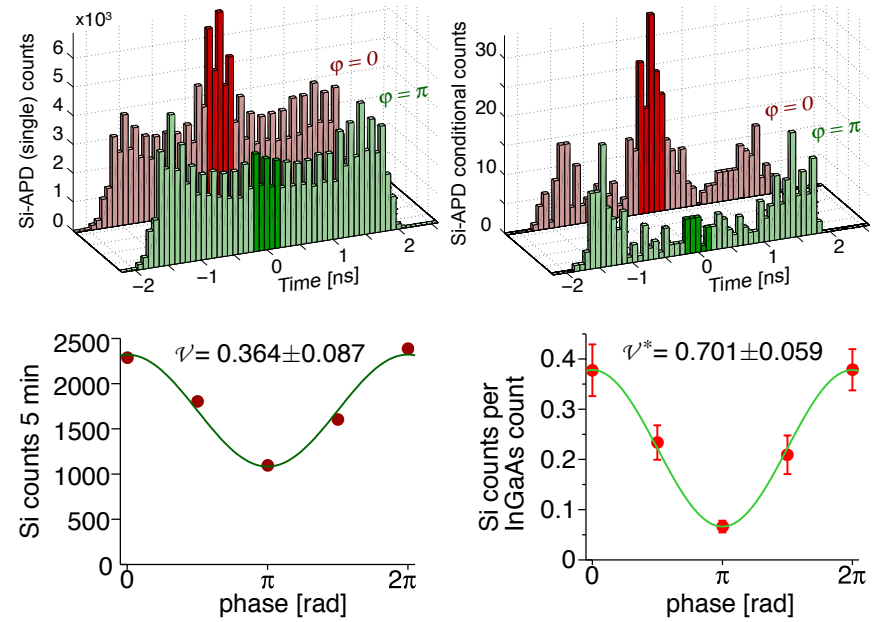

FIG. 4: (color online) Retrieval of qubits created in a superposition of early and late temporal modes. The top left figure presents histograms of single detections of the retrieved $795 \mathrm{~nm}$ photons with AFC phase settings of zero (red) and $\pi$ (green), collected during $80 \mathrm{~min}$. The top right figure shows the same histograms for conditional detections. The highlighted regions mark detection windows used to derive projection probabilities required to calculate fidelities. The lower curves show single and coincidence counts obtained for all phase settings for single detections (left) and conditional detections (right), yielding visibilities of $0.364 \pm 0.087$ and $0.701 \pm 0.059$, respectively.

slightly above 1 for the single, and above 6 for the conditional detection. In the lower part of Fig. 4 we show the normalized counts for each projection setting for the single and conditional detections. Fitting sinusoidal curves to these we derive visibilities $\mathcal{V}$, which, in turn, yield a fidelity $\mathcal{F}=(1+\mathcal{V}) / 2$ for single detections of $\mathcal{F}_{\phi}=0.682 \pm 0.020$. For conditional detections we find a significantly larger value of $\mathcal{F}_{\phi}^{*}=0.851 \pm 0.030$. These figures allow establishing an average, single detection fidelity: $\overline{\mathcal{F}} \equiv\left(\mathcal{F}_{\text {el }}+2 \mathcal{F}_{\phi}\right) / 3=0.738 \pm 0.029$. This violates the quantum classical bound [28] of $\sim 0.667$, thus verifying that our memory outperforms any classical storage protocol. However, it is below the bound of $\sim 0.833$ for an optimal universal quantum cloner 29. Harnessing the conditional detection we find $\overline{\mathcal{F}^{*}}=0.885 \pm 0.020$. This beats the quantum-classical bound by 10 standard deviations and also violates the optimal universal quantum cloner bound by 2.5 standard deviations.

To conclude, we have demonstrated storage, retrieval, and conditional detection of different time-bin qubit states using a solid-state Ti:Tm:LiNbO3 waveguide quantum memory with average fidelity $\overline{\mathcal{F}^{*}}=0.885 \pm 0.020$, which exceeds the relevant classical bounds. Operating the memory in a heralded fashion is readily achievable with high-rate APDs that have recently become commercially available. Despite our memory device's current limitations, namely efficiency, storage time, and preset recall time, the high fidelity and the wide spectral accep- tance makes our approach promising for future quantum communication schemes and quantum networks. The $\mathrm{LiNbO}_{3}$ host crystal and the waveguide structure have potential advantages in quantum memory applications such as fast electric field control of collective atomic phase evolution and, due to the resemblance with building blocks of classical integrated optical devices [30, it holds promise for simple integration with existing information technology. Furthermore, the ability to perform projection measurements using a photon-echo memory provides a simple and robust tool that might find use in other applications of quantum information processing.

We thank C. La Mela and T. Chanelière for helping in the initial stages of this work, V. Kiselyov for technical support, and NSERC, GDC, iCORE (now part of AITF), QuantumWorks, CFI and AET for financial support. D.O. thanks the Carlsberg Foundation and F.B. thanks FQRNT for support.

* These authors contributed equally.

$\dagger$ Current address: GAP-Optique, Univ. of Geneva, Rue de l'École-de-médecine 20, 1211 Geneva, Switzerland

[1] H.-J. Briegel et al., Phys. Rev. Lett. 81, 5932 (1998).

[2] N. Sangouard et al., Rev. Mod. Phys. 83(1), 33 (2011).

[3] J. Kimble, Nature 453, 1023 (2008).

[4] P. Kok et al., Rev. Mod. Phys. 79, 135 (2007).

[5] H. P. Specht et al., Nature 473, 190 (2011).

[6] H. Tanji et al., Phys. Rev. Lett. 103, 043601 (2009).

[7] W. Tittel et al., Laser \& Phot. Rev. 4, 244 (2010).

[8] A. I. Lvovsky, B.C. Sanders and W. Tittel, Nature Photonics 3, 706 (2009).

[9] M. Hosseini et al., Nature Physics 7, 794 (2011).

[10] M. Hedges et al., Nature 465, 1052 (2010).

[11] E. Saglamyurek, et al., Nature 469), 512 (2011)

[12] C. Clausen, et al., Nature 469, 508 (2011)

[13] H. Zhang et al., Nat. Photon. 5, 628 (2011).

[14] K. F. Reim et al., Phys. Rev. Lett. 107, 053603 (2011).

[15] K. Akiba et al., New J. Phys. 11, 013049 (2009).

[16] M. D. Eisaman, et al., 82(7), 071101 (2011)

[17] ID-Quantique SA, Physics today, 64, 59 (2011).

[18] N. Sinclair et al., J. Lumin. 130, 1586 (2010).

[19] M. Afzelius et al., Phys. Rev. A 79, 052329 (2009).

[20] W. Tittel and G. Weihs, Quant. Inf. Comp., 1(2), 3 (2001)

[21] C. Thiel, T. Böttger and R. Cone, J. Lumin. 131, 353 (2011).

[22] C. W. Thiel et al., J. Lumin. 130, 1598 (2010).

[23] R. R. Reibel et al., J. Lumin. 107, 103 (2004).

[24] S. A. Moiseev and B. S. Ham, Phys. Rev. A 70, 063809 (2004).

[25] H. de Riedmatten et al., Nature 456, 773 (2008).

[26] M. Hosseini et al., Nature 461, 241 (2009).

[27] S. A. Moiseev and W. Tittel, Phys. Rev. A 82, 012309 (2010).

[28] S. Massar and S. Popescu, Phys. Rev. Lett. 74, 1259 (1995).

[29] V. Bužek and M. Hillery, Phys. Rev. A 54, 1844 (1996).

[30] W. Sohler et al., Opt. Photon. News 19, 24 (2008). 